\title{
Excitation of Morphology-Dependent Resonances and Van De Hulst's Localization Principle
}

James A. Lock

Cleveland State University, j.lock@csuohio.edu

Follow this and additional works at: https://engagedscholarship.csuohio.edu/sciphysics_facpub

Part of the Physics Commons

How does access to this work benefit you? Let us know!

\section{Publisher's Statement}

This paper was published in Optics Letters and is made available as an electronic reprint with the permission of OSA. The paper can be found at the following URL on the OSA website: http://www.opticsinfobase.org/ol/abstract.cfm?URI=ol-24-7-427. Systematic or multiple reproduction or distribution to multiple locations via electronic or other means is prohibited and is subject to penalties under law.

\section{Original Citation}

Lock, James A. "Excitation of Morphology-Dependent Resonances and Van De Hulst's Localization Principle." Optics Letters 24 (1999): 427-429.

\section{Repository Citation}

Lock, James A., "Excitation of Morphology-Dependent Resonances and Van De Hulst's Localization Principle" (1999). Physics Faculty Publications. 7.

https://engagedscholarship.csuohio.edu/sciphysics_facpub/7

This Article is brought to you for free and open access by the Physics Department at EngagedScholarship@CSU. It has been accepted for inclusion in Physics Faculty Publications by an authorized administrator of EngagedScholarship@CSU. For more information, please contact library.es@csuohio.edu. 


\title{
Excitation of morphology-dependent resonances and van de Hulst's localization principle
}

\author{
James A. Lock \\ Department of Physics, Cleveland State University, Cleveland, Ohio 44115
}

Received October 30, 1998

\begin{abstract}
When a laser beam scatters from a microparticle whose shape deviates from that of a sphere, a number of partial waves of the incident beam couple to a given partial wave of the scattered and interior fields. As a result, partial-wave coupling caused by small surface irregularities of a liquid droplet provides the mechanism for exciting low-radial-order morphology-dependent resonances. (c) 1999 Optical Society of America

OCIS codes: $290.4020,290.5850$.
\end{abstract}

For scattering of a plane wave of wave number $k=$ $2 \pi / \lambda$ by a sphere of radius $a$, van de Hulst's localization principle ${ }^{1}$ was originally stated as follows: The angular dependence of the scattered light for each scattering process (i.e., reflection, transmission, etc.) in Mie theory is compared with that of ray theory. The scattered electric fields of the two theories nearly coincide for $a \gg \lambda$ if the partial wave $l$ of the incident plane wave is associated with an incident geometrical ray that has the impact parameter $\rho_{0}$ with respect to the center of the sphere:

$$
k \rho_{0}=l+1 / 2 .
$$

The association is restricted to $l<k a$ because only the rays with $\rho_{0}<a$ strike the sphere. Later, the localization principle was extended ${ }^{2,3}$ to predict that a morphology-dependent resonance (MDR) of a partial wave $l>k a$ is most efficiently excited when a focused incident beam, rather than a plane wave, passes the distance $\rho_{0}>a$ from the center of the sphere with $l$ and $\rho_{0}$ related by Eq. (1). Inasmuch as the beam classically passes the sphere by without striking it, the MDR is excited by the incident partial wave tunneling through the centrifugal barrier surrounding the sphere. ${ }^{4}$ The localization principle then was extended to the partial-wave coefficients of the focused incident beam itself., ${ }^{5,6}$ Again the relation between the partialwave number $l$ about which the incident beam coefficients are sharply peaked and the impact parameter $\rho_{0}$ of the focused beam with respect to the origin of coordinates is given by Eq. (1).

In a recent Letter ${ }^{7}$ an experiment was described whose purpose was to determine experimentally the position of a focused laser beam that most efficiently excites a MDR in a liquid droplet of nominal radius $a$. It was found that the incident beam position that most efficiently excited high-radial-order MDR's was beyond the droplet's edge, although not so far beyond as Eq. (1) predicts. For low-radial-order MDR's, however, the most efficient excitation position was found to be slightly inside the droplet's edge. This result, which apparently violates the extension of the localization principle to MDR excitation, was attributed to scattering by irregularities in the shape of the droplet's surface. These irregularities include both an overall distortion produced by hydrodynamic forces acting on the falling droplets and small-amplitude thermally induced surface capillary waves. This attribution was supported in Ref. 7 by a numerical computation of the energy inside the droplet in the MDR mode with the droplet shape modeled by

$$
r(\cos \theta)=a[1+\epsilon \cos (20 \theta)]
$$

and $\epsilon \ll 1$. For this surface shape, the beam position that most efficiently excited the $\mathrm{TE}_{97}^{1}$ resonance shifted from the prediction of Eq. (1) to slightly inside the droplet edge when $10^{-6}<\epsilon<10^{-5}$. These results illustrate that, in experimental situations, lowradial-order MDR's of liquid droplets are excited not by partial-wave tunneling but rather by a different mechanism involving scattering by surface irregularities that does not occur for spheres but that dominates when the height of the irregularities is less than a tenth of a nanometer.

The purposes of this Letter are to provide a more detailed physical mechanism for the excitation of lowradial-order MDR's in microparticles that have small surface irregularities and to show that the mechanism is consistent with the extension of the localization principle to the incident beam coefficients. The light-scattering equations of generalized Lorenz-Mie theor $\mathrm{y}^{8}$ were derived to first order in the surface perturbation from a sphere following the method of $\mathrm{Yeh}^{9}$ and Erma. ${ }^{10}$ The first-order perturbation theory results given here employ the notation of Ref. 8 and are expected to be accurate because the height of the irregularities is much smaller than the wavelength of light. The particle has refractive index $n$, and its axisymmetric surface shape is

$$
r(\cos \theta)=a[1+\epsilon f(\cos \theta)],
$$

where $\epsilon f \ll 1$. The incident focused beam propagates parallel to the $z$ axis, has electric-field half-width $w_{0}$ in its focal plane, and is decomposed into partial waves with coefficients $A_{l, m}$ and $B_{l, m}$. The Mie theory partial-wave scattering and interior amplitudes for a plane wave scattered by a sphere of radius $a$ are $a_{l}, b_{l}$ 
and $c_{l}, d_{l}$, respectively. The analogous partial-wave scattering and interior amplitudes for a focused beam scattered by a particle with the surface shape of Eq. (3) are $\alpha_{l, m}, \beta_{l, m}, \gamma_{l, m}$, and $\delta_{l, m}$. These amplitudes, to first order in $\epsilon$, are given by

$$
\begin{aligned}
\alpha_{l, m}= & a_{l} A_{l, m}-i \epsilon c_{l} \sum_{l^{\prime}}\left[l^{\prime}\left(l^{\prime}+1\right) c_{l^{\prime}} A_{l^{\prime}, m} R_{l^{\prime}, l} I_{l^{\prime}, l, m}^{2} /(k a)^{2}\right. \\
& \left.+c_{l^{\prime}} A_{l^{\prime}, m} U_{l^{\prime}, l} I_{l^{\prime}, l, m}^{1}-d_{l^{\prime}} B_{l^{\prime}, m} S_{l^{\prime}, l} J_{l^{\prime}, l, m}\right], \\
\beta_{l, m}= & b_{l} B_{l, m}-i \epsilon d_{l} \sum_{l^{\prime}}\left(d_{l^{\prime}} B_{l^{\prime}, m} R_{l^{\prime}, l} I_{l^{\prime}, l, m}^{1}\right. \\
& \left.-c_{l^{\prime}} A_{l^{\prime}, m} T_{l^{\prime}, l} J_{l^{\prime}, l, m}\right), \\
\gamma_{l, m}= & c_{l} A_{l, m}+i \epsilon c_{l} \sum_{l^{\prime}}\left[l^{\prime}\left(l^{\prime}+1\right) c_{l^{\prime}} A_{l^{\prime}, m} W_{l^{\prime}, l} I_{l^{\prime}, l, m}^{2} / n(k a)^{2}\right. \\
& \left.+c_{l^{\prime}} A_{l^{\prime}, m} Z_{l^{\prime}, l} I_{l^{\prime}, l, m}^{1}-d_{l^{\prime}} B_{l^{\prime}, m} X_{l^{\prime}, l} J_{l^{\prime}, l, m}\right], \\
\delta_{l, m}= & d_{l} B_{l, m}+i \epsilon d_{l} \sum_{l^{\prime}}\left(d_{l^{\prime}} B_{l^{\prime}, m} W_{l^{\prime}, l} I_{l^{\prime}, l, m}^{1}\right. \\
& \left.-c_{l^{\prime}} A_{l^{\prime}, m} Y_{l^{\prime}, l} J_{l^{\prime}, l, m}\right) .
\end{aligned}
$$

In Eqs. (4)-(7) the radial matrix elements are

$$
\begin{aligned}
& R_{l^{\prime}, l}=Q_{l^{\prime}, l} \psi_{l}(n k a) \psi_{l^{\prime}}(n k a), \\
& S_{l^{\prime}, l}=Q_{l^{\prime}, l} \psi_{l}^{\prime}(n k a) \psi_{l^{\prime}}(n k a), \\
& T_{l^{\prime}, l}=Q_{l^{\prime}, l} \psi_{l}(n k a) \psi_{l^{\prime}}^{\prime}(n k a), \\
& U_{l^{\prime}, l}=Q_{l^{\prime}, l} \psi_{l}^{\prime}(n k a) \psi_{l^{\prime}}^{\prime}(n k a), \\
& W_{l^{\prime}, l}=Q_{l^{\prime}, l} \xi_{l}^{(1)}(k a) \psi_{l^{\prime}}(n k a), \\
& X_{l^{\prime}, l}=Q_{l^{\prime}, l} \xi_{l}^{(1) \prime}(k a) \psi_{l^{\prime}}(n k a), \\
& Y_{l^{\prime}, l}=Q_{l^{\prime}, l} \xi_{l}^{(1)}(k a) \psi_{l^{\prime}}^{\prime}(n k a), \\
& Z_{l^{\prime}, l}=Q_{l^{\prime}, l} \xi_{l}^{(1) \prime}(k a) \psi_{l^{\prime}}^{\prime}(n k a),
\end{aligned}
$$

where the outgoing Riccati-Bessel function is $\xi_{l}^{(1)}=$ $\psi_{l}+i \chi_{l}$ and

$Q_{l^{\prime}, l}=k a\left(n^{2}-1\right) i^{l^{\prime}-l} l(l+1)\left(2 l^{\prime}+1\right) / l^{\prime}\left(l^{\prime}+1\right)(2 l+1)$.

The angular matrix elements are

$$
\begin{aligned}
I_{l^{\prime}, l, m}^{1}= & \frac{(2 l+1)(l-|m|) !}{2 l(l+1)(l+|m|) !} \int_{0}^{\pi} \sin \theta \mathrm{d} \theta f(\cos \theta) \\
& \times\left(\tau_{l^{\prime}}^{|m|} \tau_{l}^{|m|}+m^{2} \pi_{l^{\prime}}^{|m|} \pi_{l}^{|m|}\right) \\
I_{l^{\prime}, l, m}^{2}= & \frac{(2 l+1)(l-|m|) !}{2(l+|m|) !} \int_{0}^{\pi} \sin \theta \mathrm{d} \theta f(\cos \theta) \\
& \times P_{l^{\prime}}^{|m|} P_{l}^{|m|}, \\
J_{l^{\prime}, l, m}= & \frac{(2 l+1)(l-|m|) !}{2 l(l+1)(l+|m|) !} m \int_{0}^{\pi} \sin \theta \mathrm{d} \theta f^{\prime}(\cos \theta) \\
& \times P_{l^{\prime}}^{|m|} P_{l}^{|m|},
\end{aligned}
$$

where $P_{l}^{|m|}$ are associated Legendre polynomials and $\tau_{l}^{|m|}$ and $\pi_{l}^{|m|}$ are the angular functions of generalized Lorenz-Mie theory. ${ }^{8}$ When the beam focal waist is located at $\rho_{0} \cos \phi_{0} \hat{u}_{x}+\rho_{0} \sin \phi_{0} \hat{u}_{y}$ with $\rho_{0} \sim a$, the incident beam partial-wave coefficients for a focused Gaussian beam and $l \gg 1$ are given by ${ }^{8}$

$$
\begin{aligned}
A_{l, m} \approx & K_{l, m}^{\max }\left[(l+1 / 2) / k \rho_{0}\right]^{1 / 2} \cos \phi_{0} \\
& \times \exp \left[-\left(k \rho_{0}-l-1 / 2\right)^{2} /\left(k w_{0}\right)^{2}\right], \\
B_{l, m} \approx & K_{l, m}^{\max }\left[(l+1 / 2) / k \rho_{0}\right]^{1 / 2} \sin \phi_{0} \\
& \times \exp \left[-\left(k \rho_{0}-l-1 / 2\right)^{2} /\left(k w_{0}\right)^{2}\right],
\end{aligned}
$$

where

$$
K_{l, \pm m}^{\max }=(-i)^{|m|-1} k w_{0} \exp \left(\mp i|m| \phi_{0}\right) / \pi^{1 / 2}(l+1 / 2)^{|m|}
$$

is the maximum value of $A_{l, m}$ and $B_{l, m}$, which occurs when $l$ satisfies Eq. (1).

Equations (4)-(7) have a pleasing physical interpretation. The first term of each equation describes scattering by a sphere of radius $a$, where a given incident beam partial wave $l$ couples to only the same partial wave $l$ of the scattered and interior fields. The remaining terms describe scattering to first order in $\epsilon$ by the surface irregularity $a \epsilon f(\cos \theta)$, where a number of different incident beam partial waves $l^{\prime}$ couple to a given partial wave $l$ of the scattered and interior fields. In the language of quantum mechanics, some of the angular momentum of the incident partial wave is taken up by a Fourier component of the irregularity, producing the different angular momenta of the scattered and interior fields. For the Fourier component $f(\cos \theta)=\cos (p \theta)$, the range of the partial-wave coupling is $l-p \leq l^{\prime} \leq l+p$, the angular matrix elements $I_{l^{\prime}, l, m}^{1}$ and $I_{l^{\prime}, l, m}^{2}$ vanish when $l^{\prime}-l$ is odd (even) if $p$ is even (odd), and $J_{l^{\prime}, l, m}$ vanishes when $l^{\prime}-l$ is even (odd) if $p$ is even (odd) as a 
result of the algebra of associated Legendre polynomials. Numerical computations with a number of different values of $l, l^{\prime}, p$, and $|m|$ showed that, for $f(\cos \theta)=\cos (p \theta)$, the value of $I_{l^{\prime}, l, m}^{1}$ was $\sim O(1)$ only when $l^{\prime}=l-p$ or $l^{\prime}=l+p$. Otherwise $I_{l^{\prime}, l, m}^{1} \sim O\left(10^{-3}\right)$ for all other allowed values of $l^{\prime}$. Thus a given Fourier component of the surface irregularity strongly couples two incident beam partial waves to a given partial wave of the scattered and interior fields. Because the surface irregularity is assumed to be axisymmetric, the $z$ component of the angular momentum is conserved; i.e., the azimuthal mode number $m$ of the incident beam, the scattered fields, and the interior fields is identical. Scattering by the surface irregularity also has both a polarization-preserving component proportional to $I_{l^{\prime}, l, m}^{1}$ and $I_{l^{\prime}, l, m}^{2}$ and a crosspolarized component proportional to $J_{l^{\prime}, l, m}$.

In Ref. 7, the partial-wave $l=97$ resonance $\mathrm{TE}_{97}^{1}$ in a microparticle with the surface shape of Eq. (2) and $n=1.332$ was calculated for an incident focused Gaussian beam with $\lambda=0.5145 \mu \mathrm{m}$ and $\phi_{0}=\pi / 2$ such that $A_{l, m}=0$ and $B_{l, m} \neq 0$. The energy inside the particle in the MDR with azimuthal mode number $m$ is proportional to $\left|\delta_{97, m}\right|^{2}$. If the incident beam is positioned beyond the edge of the particle as in Eq. (1) such that $k \rho_{0}=97.5$, and the beam is tightly focused so $B_{l, m}$ rapidly decreases as a function of $l$, the interior partial-wave amplitude can be approximated by the first term of Eq. (7):

$$
\delta_{97, m} \approx d_{97} K_{97, m}^{\max },
$$

and MDR excitation occurs by means of tunneling. If instead the incident beam is positioned such that $k \rho_{0}=77.5$, and the beam is again tightly focused so $B_{l, m}$ rapidly decreases as a function of $l^{\prime}$, the interior partial-wave amplitude of Eq. (7) is

$$
\delta_{97, m} \approx i \epsilon d_{97} d_{77} K_{77, m}^{\max } W_{77,97} I_{77,97, m}^{1},
$$

and MDR excitation occurs through angular momentum coupling. If $\epsilon$ is large enough, the magnitude of $\delta_{97, m}$ in relation (24) becomes greater than that in relation (23). As the size parameter of the particle is $k a \approx 78.558$ at the $\mathrm{TE}_{97}^{1}$ resonance, the partial wave $l^{\prime}=77$ corresponds to the focused incident beam lying just inside the droplet surface through the extension of the localization principle to the incident beam partialwave coefficients. Thus, if $\epsilon$ is large enough, positioning the beam just inside the edge of the particle so light enters it by refraction and using the surface shape ir- regularity to couple the incident partial wave to the resonant partial wave excite the MDR more than do positioning the beam the appropriate distance beyond the edge of the particle and having the MDR be excited by tunneling.

Numerical computations showed that, for the values of the parameters given above, $\left|d_{77} W_{77,97}\right|=8.1 \times 10^{5}$ and $I_{77,97, m}^{1} \sim 0.3$ for small $|m|$. Also, $K_{77, m}^{\max }$ and $K_{97, m}^{\max }$ are comparable for small $|m|$. Thus, when $\epsilon$ is larger than $\sim 4 \times 10^{-6}$, corresponding to a surface roughness of $\sim 0.03 \mathrm{~nm}$, the partial-wave coupling mechanism dominates the tunneling mechanism in exciting the $\mathrm{TE}_{97}^{1}$ resonance. This crossover value of $\epsilon$ agrees with the results shown in Fig. 5 of Ref. 7. Generalization of the partial-wave coupling mechanism to other Fourier components of the surface shape, other resonances, other propagation directions of the incident beam, and nonaxisymmetric irregularities is straightforward.

In conclusion, although the tunneling mechanism excites low-radial-order MDR's in a sphere, it is the angular momentum coupling mechanism that excites these resonances in experimental situations with liquid droplets because the heights of the thermally induced capillary waves upon the surface of a droplet are of the order of a few tenths of a nanometer, and the capillary waves' spatial frequency spectrum is quite broad.

This study was supported in part by NASA grant NCC3-521. The author thanks the authors of Ref. 7 for making a preprint of their manuscript available before publication.

\section{References}

1. H. C. van de Hulst, Light Scattering by Small Particles (Dover, New York, 1981), pp. 208-214.

2. S. C. Hill and R. E. Benner, in Optical Effects Associated with Small Particles, P. W. Barber and R. K. Chang, eds. (World Scientific, Singapore, 1988), p. 40.

3. J. P. Barton, D. R. Alexander, and S. A. Schaub, J. Appl. Phys. 65, 2900 (1989).

4. H. M. Nussenzveig, Comments At. Mol. Phys. 23, 175 (1989).

5. G. Grehan, B. Maheu, and G. Gouesbet, Appl. Opt. 25, 3539 (1986).

6. B. Maheu, G. Grehan, and G. Gouesbet, Opt. Commun. 70, 259 (1989).

7. H.-B. Lin, J. D. Eversole, A. J. Campillo, and J. P. Barton, Opt. Lett. 23, 1921 (1998).

8. J. A. Lock, Appl. Opt. 34, 559 (1995).

9. C. Yeh, Phys. Rev. 135, A1193 (1964).

10. V. Erma, Phys. Rev. 173, 1243 (1968), 176, 1544 (1968), 179, 1238 (1969). 